\title{
The Type Specimens of Sucking Lice (Anoplura) Deposited in the Entomological Collection of Instituto Oswaldo Cruz, Rio de Janeiro, RJ, Brazil
}

\author{
Margareth Cardozo-de-Almeida/ ${ }^{+}$, Pedro Marcos Linardi* , Jane Costa
}

Núcleo de Informatização, Coleção Entomológica, Departamento de Entomologia, Instituto Oswaldo Cruz, Av. Brasil 4365, 21045-900 Rio de Janeiro, RJ, Brasil *Departamento de Parasitologia, ICB, Universidade

Federal de Minas Gerais, Caixa Postal 486, 30161-970 Belo Horizonte, MG, Brasil

This study presents a list of 34 Anoplura type specimens deposited in the Werneck Collection of Instituto Oswaldo Cruz, Rio de Janeiro, RJ, Brazil. It includes 18 holotypes, 16 allotypes, 88 paratypes and 10 neotypes, distributed among the genera: Enderleinellus, Fahrenholzia, Haematopinus, Hoplopleura, Linognathus, Microthoracius, Pecaroecus, Polyplax and Pterophthirus. The types are related according to their respective data and literature.

Key words: type specimens - Anoplura - sucking lice - Werneck Collection - Entomological Collection Instituto Oswaldo Cruz - Brazil

The Entomological Collection of the Instituto Oswaldo Cruz (IOC) includes approximately 1.2 million specimens, representing all the orders, being one of the most expressive of Latin America. It is constituted by a general collection and by the Herman Lent Collection of Hemiptera, which presents specimens of Reduviidae and mainly of subfamily Triatominae, the vectors of Chagas disease. It also includes other historical collections such as Adolpho Lutz, Cesar Pinto, Costa Lima, Henrique Aragão, Lauro Travassos, Otavio Mangabeira Filho, FL Werneck and JF Zikan. These historical collections were closed after the death of their dedicated researchers, being a rich historical and scientific holding that integrates specimens captured by the researchers themselves during the first expeditions undertaken in Brazil since 1900.

The historical Werneck Collection includes specimens of the orders Anoplura, Hemiptera, Mallophaga and Siphonaptera, deriving from captures undertaken by Werneck and other researchers, performed in 1911-1960, in Brazil and other countries, as well as by donations to IOC, provided by international and Brazilian institutions. In the

Work performed with support of Papes-Fiocruz Project and presented on the I Bienal de Pesquisa do Instituto Oswaldo Cruz.

*Research fellow CNPq.

${ }^{+}$Corresponding author. Fax: +55-21-2909339. E-mail: margarethalmeida@hotmail.com

Received 29 December 1998

Accepted 26 March 1999
Werneck Collection, there are 1,246 slides of Anoplura containing 120 species, from which 34 are type specimens and other 88 paratypes and 10 neotypes, summing 125 slides.

In attention to the determination 72D of the International Code of Zoological Nomenclature, which says that "every institution in which types are deposited should publish lists of type-material in its possession or custody", some surveys on the type species of various groups have been carried out in the entomological collections of IOC: triatomines (Gonçalves et al. 1994), culicides (Marchon-Silva et al. 1996) and specimens of superfamily Apoidea (Ferraz 1997). Intending update and disseminate the content of the Entomological Collection of IOC, the present study addresses the type specimens deposited in the Werneck Collection of this institution.

Anoplura are ectoparasitic insects, exclusively of mammals, presenting an obligatory hematophagy in nymphal and adult stages, commonly known as sucking lice. Their parasitological importance derives from the actions they exert on hosts as infesting agents (Nelson et al. 1977), or as vectors diseases: to human beings - louse-borne typhus (epidemic typhus), trench fever, relapsing fever, transmitted by Pediculus humanus (reviewed by Weidhaas \& Gratz 1982) - or among rodents: murine typhus (endemic typhus), transmitted by species of Polyplax and Hoplopleura (Traub et al. 1978).

Given their parasitic specificity, Anoplura are of fundamental importance to studies on co-evolution with their respective hosts (Hopkins 1949, Vanzolini \& Guimarães 1955, Kim 1988, Barker 1994). Until now, 33 species were recorded in Bra- 
zil, including 17 cosmopolitan (Durden \& Musser 1994). Among Brazilian or Neotropical species, eight were described by Werneck (1932a, b, 1933, 1937, 1942), four by Ferris $(1916,1921)$, two by Linardi et al. (1984a, b), one by Guimarães (1950) and another by Johnson (1972). According to the geographical distribution presented by Durden and Musser (1994), we might consider the possibility of other 10 species also occurring in Brazil.

The methodology applied here was the same used by Marchon-Silva et al. (1996). Besides the information about type specimens we added the name of the host on which the material was collected.

Family Enderleinellidae

Genus Enderleinellus Fahrenholz, 1912

- brasiliensis Werneck, 1937. Enderleinellus. (118) Holotype 1 male (447). Allotype 1 female (448). Paratypes 2 females $(449,450)$; Sciurus aestuans; Abaeté, Pará, Brazil; 1936.

- urosciuri Werneck, 1937. Enderleinellus. (766) Holotype 1 male (1312). Allotype 1 female (1313). Paratypes 4 females (1314 - 1317); Urosciurus igniventris; Acajutuba, Rio Negro, Amazonas, Brazil.

Family Haematopinidae

Genus Haematopinus Leach, 1815

- acuticeps Ferris, 1933. Haematopinus. (696) Paratype 1 female (1184); Hippotigris burchelli; Mpwapwa, Tankanica Territory (=Tanzânia); 11II-1931.

- gorgonis Werneck, 1952. Haematopinus. (2911) Paratype 1 female (3618); Gorgon taurinus albojabatus; Ukerewe Peninsula, Tanganyika (=Tanzânia); GHE Hopkins coll., X-1944.

- phacochoeri meinertzhageni Werneck, 1952. Haematopinus. $(2780,2781)$ Holotype 1 male (4064). Allotype 1 female (4065). Paratypes $1 \mathrm{fe}-$ male (3500) and 2 males (3501, 3502); Hylochoerus meinerzthageni meinertzhageni; near Lukiri, Buhwezu Co., Ankole, Uganda; EU Hines coll., VI-1946. Synonym of Haematopinus meinertzhageni Werneck, 1952. Durden \& Musser, 1994 : 16 (syn.).

Family Hoplopleuridae

Genus Hoplopleura Enderlein, 1904

- affinis argentina Werneck, 1937. Hoplopleura. (831) Holotype 1 female (1653). Paratypes 2 females (1654, 1655); Reithrodon sp. (wild rat); Argentina; D Ponte coll. Synonym of Hoplopleura argentina Werneck, 1937. Ferris, 1951 : 133 (syn.). - brasiliensis Werneck, 1937. Hoplopleura. (23) Holotype and Allotype 1 female (186) and 1 male (186). Paratypes 3 females $(187,189,190)$ and 3 males (190, 192, 193); (wild rat); Veadeiro, Goiás, Brazil.

- capensis Werneck, 1954. Hoplopleura. (3334) Paratype 1 female (3917); Tatera sp.; Seepduik, Hay District, Cape Province, South Africa; South African Biol. Survey coll., 8-XII-1943.

- disgrega chilensis Werneck, 1937. Hoplopleura. (957) Holotype 1 female (1951). Allotype 1 male (1952). Paratypes 4 females (1953 - 1956) and 3 males (1957 - 1959); Octodon degus (rat); Chile. Synonym of Hoplopleura chilensis Werneck, 1937. Ferris, $1951: 134$ (syn.).

- fonsecai Werneck, 1933. Hoplopleura. (441) Holotype 1 female (916). Allotype 1 male (917). Paratypes 4 females (918 - 921) and 1 male (922); Oxymycterus judex (wild rat); Humboldt, Sta. Catarina, Brazil; W Etrhardt coll., 5-VII-1918.

- malabarica Werneck, 1954. Hoplopleura. (3384) Paratypes 4 females (3918) and 1 male (3918); Bandicota malabarica; Colombo, Ceylão (=Sri Lanka); CD Radford coll., 28-VII-1944.

- multilobata Werneck, 1954. Hoplopleura. (3295) Holotype 1 female (3893); Proechimys (Proechimys) iheringi; Santa Tereza, Espírito Santo, Brazil; 22-VI-1940.

- travassosi Werneck, 1932. Hoplopleura. (452) Holotype 1 female (908). Allotype 1 male (909). Paratypes 2 females (906, 907); Oryzomys flavescens (wild rat); Humboldt, Sta. Catarina, Brazil; W Etrhardt coll., 1-VIII-1918.

Genus Pterophthirus Ewing, 1923

- imitans Werneck, 1942. Pterophthirus. (1565) Holotype 1 male (2764). Allotype 1 female (2761). Paratypes 2 females (2762, 2763); Cavia aperea; Santo Amaro, São Paulo, Brazil; LR Guimarães coll., 6-I-37.

- wernecki Guimarães, 1950. Pterophthirus. (2759) Paratypes 1 female (3446) and 1 male (3447); Proechimys iheringi iheringi; Boracéia, São Paulo, Brazil; LT Filho coll.

Family Linognathidae

Genus Linognathus Enderleinus, 1905

- contractus Werneck, 1959. Linognathus. (3980) Paratypes 1 female (4026) and 2 females (flask 368, support 37); Ourebia ourebi; Reserva de caça da Cameia, Angola; AB Machado coll., 3-XII-1954. - fractus Ferris, 1932. Linognathus. (3964) Holotype 1 male (4066). Allotype 1 female (4067). Paratypes 4 females (4044 - 4047), 3 males (4048 - 4050) and genitalia of male (4051, 4052); Strepsiceros s. strepsiceros; Zululandia, South Africa; R. du Toit coll.

- ourebiae Werneck, 1959. Linognathus. (3984) Paratypes of both sexs (flask 369, support 37); Ourebia ourebi; Reserva de caça da Cameia, 
Angola; AB Machado coll., 3-XII-1954.

- raphiceri Fiedler \& Stampa, 1956. Linognathus. (3992) Holotype 1 female (4068). Allotype 1 male (4069). Paratypes 2 females $(4040,4041)$ and 2 males (4042, 4043); Raphicerus campestris; Zululandia, África do Sul; R. du Toit coll.

- taeniotrichus Werneck, 1937. Linognathus. (100) Holotype 1 female (421). Allotype 1 male (422). Paratypes 2 females $(423,424)$ and 1 male (425); Cerdocyon guaraxa (fox); São Bernardo das Russas, Ceará, Brazil; 1936.

- vulpis Werneck, 1952. Linognathus. (2909) Holotype 1 male (3613). Allotype 1 female (3612); Vulpes rüppellii bengalensis (fox); Kurackee, Paquistão; FL Werneck coll.

\section{Genus Solenopotes}

- hologastrus Werneck, 1937. Linognathus. (161) Holotype 1 female (634). Allotype 1 male (635). Paratypes 1 female (636) and 1 male (637); Gorgon taurinus; Grootfonteien, South West Africa; 19VI-1933. Synonym of Solenopotes hologastrus Werneck, 1937. Durden \& Musser, 1994 : 45 (syn.).

Family Microthoraciidae

Genus Microthoracius Fahrenholz,1916

- cameli (Linnaeus, 1758). Microthoracius. (668) Neotypes 5 females (1122 - 1126) and 5 males (1127 - 1131); Camelus dromedarius (camel); Hassi Bahbah, Depart Alger, Algeria; A Lepigre coll., V-1934.

- mazzai Werneck, 1932. Microthoracius. (50) Holotype 1 female (239). Allotype 1 male (240). Paratypes 8 females (241 - 248), 3 males (249 251), 5 nymphs (252 - 256), spermatheca (257); Auchenia llama; Sta. Catalina, Prov. Jujuy, Argentina; S Mazza coll.

- minor Werneck, 1935. Microthoracius. (808) Holotype 1 female (1514). Allotype 1 male (1515); Lama pacos; Abra Pampa, Prov. de Jujuy, Argentina; S Mazza coll.

Family Pecaroecidae

Genus Pecaroecus Babcock \& Ewing, 1938

- javalli Babcock \& Ewing, 1938. Pecaroecus. (2288) Paratypes (Cotypes) 1 female (2855) and 1 male (2856); (Peccary); Juno, Texas, Estados Unidos; OG Babcock coll., 29-I-1932.

Family Polyplacidae

Genus Fahrenholzia Kellogg \& Ferris, 1915

- schwartzi Werneck, 1952. Fahrenholzia. (2880) Holotype 1 male (3568). Allotype 1 female (3569). Paratypes 1 female (3571) and 1 male (3570); Heteromys anomalus anomalus; Sierra Maestra, Est. de Aragua, Venezuela; Amberson \& Schwarz colls.
Genus Polyplax Enderlein, 1904

- grammomydis Werneck, 1953. Polyplax. (2970) Paratypes 2 females $(3684,3685)$ and 2 males (3687, 3688); Grammomys dolichurus; Gowies Kloof, Albany, Prov. do Cabo, África do Sul; 2-II1940.

\section{Genus Galeophthirus}

- caviae Werneck, 1934. Eulinognathus. (629) Holotype 1 female (1141). Allotype 1 male (1145). Paratypes 3 females and 3 males (1142-1144, 11461148); Galea leucoblephara; Perico, Prov. Jujuy, Argentina; S Mazza coll., III-1934. Synonym of Galeophthirus caviae Werneck, 1934. In Ferris, 1951 (syn.).

\section{DISCUSSION}

According to Kim and Ludwig (1978), the Neotropical region concentrates $11 \%$ of the whole world anoplurofauna. Presently, 15 families are known, and they are distributed in 49 genera and 532 species (Durden \& Musser 1994), from which $120(22.6 \%)$ are represented in the Werneck Collection of IOC. The contribution of Werneck for the improvement of our knowledge on Anoplura is highly significant, once he had described 34 species $(6.4 \%)$, from those recorded in the world as a whole. It must be also stressed that until the middle of the century, while only 255 species were known (Ferris 1951), Werneck had already described 21 species from several regions of the world. Consequently, the result of this effort, now documented in the Werneck Collection of IOC, is indispensable for the modern taxonomy, noticing that through the comparative study of the characteres, the reference colletions represent nowadays a starting point to new investigations (Martins 1983).

In honour to his work, the author's name was given to one genera and two species, by different authors: Werneckia, by Ferris (1951); Pterophthirus wernecki, by Guimarães (1950) and Eulinognathus wernecki, by Castro and Cicchino (1986).

\section{AKNOWLEDGMENTS}

To Dr Rubens Pinto de Mello, for the revision of the manuscript and appropriate suggestions. To Ana Paula Rufino Amaro for the bibliographic survey and the maintenance of the Werneck Collection material. To Ronaldo Ribeiro and Veronica Marchon-Silva for storing the collection data.

\section{REFERENCES}

Barker SC 1994. Phylogeny and classification, origins, and evolution of host associations of lice. Int $J$ Parasit 24: 1285-1291.

Castro D del C, Cicchino AC 1986. Contribución al conocimiento del genero Eulinognathus Cummings, 1916 (Anoplura, Polyplacidae) parasitas de 
Ctenomyidae (Rodentia, Octodontoidea). Rev Bras Entomol 30: 375-382.

Durden LA, Musser GG 1994. The sucking lice (Insecta, Anoplura) of the world: a taxonomic checklist with records of mammalian hosts and geographical distributions. Bull Amer Mus Nat Hist 218: 1-90.

Ferraz MV 1997. The type specimens of Apoidea (Hymenoptera) deposited in the Entomological Collection of Instituto Oswaldo Cruz, Rio de Janeiro, RJ, Brazil. Mem Inst Oswaldo Cruz 92: 353-356.

Ferris GF 1916. A catalogue and host list of the Anoplura. Proc Calif Acad Sci 6: 129-213.

Ferris GF 1921. Contributions toward a monograph of the sucking lice, Part II. Stanford Univ Publ Univ Ser Biol Sci 2: 52-133.

Ferris GF 1951. The sucking lice. Mem Pac Coast Entomol Soc 1: 1-320.

Gonçalves TCM, Almeida MD, Lent H, Jurberg J 1994. Lista dos exemplares-tipos de Triatomíneos depositados na Coleção Entomológica do Instituto Oswaldo Cruz, Rio de Janeiro (Hemiptera : Reduviidae). Mem Inst Oswaldo Cruz 88: 327-333.

Guimarães LR 1950. Sôbre uma nova espécie de Pterophthirus Ewing, 1923 (Anoplura). Pap Av Dep Zool S Paulo 9: 83-88.

Hopkins GHE 1949. The host-associations of the lice of mammals. Proc Zool Soc London 119: 387-604.

Johnson PT 1972. Sucking lice of Venezuelan rodents, with remarks on related species (Anoplura). Brigham Young Univ Sci Bull Biol Ser 17: 1-62.

Kim KC 1988. Evolutionary parallelism in Anoplura and eutherian mammals, p. 91-114. In MW Service, Biosystematics of Haematophagous Insects, Clarendon Press, Oxford.

Kim KC, Ludwig HW 1978. The family classification of the Anoplura. Syst Ent 3: 249-284.

Linardi PM, Teixeira VP, Botelho JR 1984a. Hoplopleura minasensis sp. n. de Minas Gerais, Brasil (Anoplura: Hoplopleuridae). Rev Brasil Biol 44: 525-531.

Linardi PM, Teixeira VP, Botelho JR 1984b. Hoplopleura imparata sp. n. de Minas Gerais e notas sobre outras espécies brasileiras de Hoplopleura (Anoplura: Hoplopleuridae). Rev Brasil Biol 44: 533-539.

Marchon-Silva V, Lourenço-de-Oliveira R, Almeida MD, Silva-Vasconcelos A, Costa J 1996. The type specimens of mosquitoes (Diptera, Culicidae) deposited in the Entomological Collection of the Instituto Oswaldo Cruz, Rio de Janeiro, Brazil. Mem Inst Oswaldo Cruz 91: 471-478.

Martins UR 1983. Coleção taxonômica, p. 1-30. In N Papavero, Fundamentos Práticos de Taxonomia Zoológica: Coleções, Bibliografia, Nomenclatura, Museu Paraense Emílio Goeldi/Conselho Nacional de Desenvolvimento Científico e Tecnológico, Belém.

Nelson WA, Bell JF, Clifford CM, Keirans JE 1977. Interaction of ectoparasites and their hosts. $\mathrm{J} \mathrm{Med}$ Entomol 13: 389-428.

Traub RT, Wisseman Jr MD, Farhang-Azad A 1978. The ecology of murine typhus - a critical review. Trop Dis Bull 75: 237-317.

Vanzolini PE, Guimarães LR 1955. South American land mammals and their lice. Evolution 9: 345-347.

Weidhaas DE, Gratz NG 1982. Lice. WHO/VBC/82.858, Geneve 1-10.

Werneck FL 1932a. Nova espécie de Anoplura. Mem Inst Oswaldo Cruz 26: 235.

Werneck FL 1932b. Sobre uma espécie nova de Hoplopleura (nota previa). Rev Med Cirurg Brasil 12: 345-346.

Werneck FL 1933. Sobre duas espécies de Anoplura encontradas em ratos sylvestres do Brasil. Mem Inst Oswaldo Cruz 27: 405-415.

Werneck FL 1934. Notas para o estudo da Ordem Anoplura. Mem Inst Oswaldo Cruz 29: 179-187.

Werneck FL 1937. Algumas espécies e subespécies novas de Anoplura. Mem Inst Oswaldo Cruz 32: 391410.

Werneck FL 1942. Pterophthirus imitans n. sp. Rev Bras Biol 2: 317-320. 